\title{
Experiências no Uso da Metodologia Coding Dojo nas Disciplinas Básicas de Programação de Computadores em um Curso Interdisciplinar do Ensino Superior
}

\author{
Carlos S. S. Marinho ${ }^{1}$, Leonardo O. Moreira ${ }^{1}$, Emanuel F. Coutinho ${ }^{1}$, \\ Gabriel A. L. Paillard ${ }^{1}$, Ernesto T. Lima Neto ${ }^{1}$ \\ ${ }^{1}$ Instituto Universidade Virtual (UFC Virtual) \\ Universidade Federal do Ceará (UFC) - Fortaleza, CE - Brasil \\ sergio.marinho94@gmail.com, \\ \{leoomoreira, emanuel, gabriel, ernesto\}evirtual.ufc.br
}

\begin{abstract}
The Coding Dojo is a methodology that aims to improve the knowledge in the area of algorithms and computer programming through collaborative learning, employing aspects of challenges and interaction. Thus, several studies have adopted the Coding Dojo methodology to assist the teaching/learning in higher education disciplines involving computer programming issues. However, such studies have used the Coding Dojo in courses more focused on the computing area and with public more focused on the sciences, engineering and computing area. This work aims to investigate the adoption of Coding Dojo methodology on a course of interdisciplinary nature, where not all participants are fully of the sciences, engineering and computing area.
\end{abstract}

Resumo. Coding Dojo é uma metodologia que visa o aperfeiçoamento do conhecimento na área de algoritmos e programação de computadores por meio da aprendizagem colaborativa, empregando aspectos de desafios e interação. Com isso, diversos trabalhos tem adotado a metodologia Coding Dojo para auxiliar o processo de ensino/aprendizagem em disciplinas do ensino superior que envolvem assuntos de programação de computadores. No entanto, tais trabalhos tem utilizado o Coding Dojo em cursos mais voltados para a área de computação e com públicos mais voltados para a área das ciências, engenharias e computação. Este trabalho tem como objetivo investigar a adoção da metodologia Coding Dojo em um curso de natureza interdisciplinar, onde nem todos os participantes são totalmente da área das ciências, engenharias e computação.

\section{Introdução e Motivação}

O curso de Sistemas e Mídias Digitais da Universidade Federal do Ceará (UFC) tem a finalidade de formar bacharéis com conhecimentos especializados em duas grandes áreas: Sistemas Multimídia e Mídias Digitais. O curso é de natureza interdisciplinar e objetiva a formação de profissionais com múltiplos saberes, permitindo uma ampla visão de seu campo de atuação. Por meio desta interdisciplinaridade, o curso de Sistemas e Mídias Digitais formam profissionais com concepção humanística, técnica e científica. Essa interseção de conhecimentos é uma proposta diferencial que o curso de Sistemas e Mídias 
Digitais visa oferecer, como profissionais, para a sociedade. De acordo com estudos feitos sobre a evasão no curso de Sistemas e Mídias Digitais, uma parcela de alunos mostraram uma certa resistência e dificuldade nas disciplinas básicas de programação de computadores. Além disso, constatou-se que existe um índice considerável de reprovações nestes primeiros contatos com as disciplinas básicas de programação de computadores. Alguns alunos recém-ingressos, que procuram uma formação mais humanística ou artística, ressaltam a não importância do conhecimento na programação de computadores em suas atividades profissionais ou até mesmo acadêmicas.

As disciplinas de programação de computadores, em geral, são ministradas por meio de aulas expositivas e laboratoriais. Diante disso, surgem dois desafios que os professores enfrentam no decorrer das aulas. O primeiro é manter a atenção e motivação dos alunos durante as aulas expositivas, pois a programação de computadores é uma atividade de raciocínio, concentração e criação [Carmo and Braganholo 2012]. O segundo desafio é oriundo da mudança no contexto universitário onde há pressões para aumentar o número de ingressos no ensino superior [Delgado et al. 2012]. Com isso, acarretando em turmas cheias e heterogêneas, dificultando o papel do professor ao orquestrar tal conteúdo para um grande quantitativo de alunos e que possuem diferentes níveis de conhecimento. Diante desses desafios, acredita-se que a adoção de uma metodologia colaborativa que empregue aspectos diferentes das aulas tradicionais pode contornar os problemas que foram apontados nas disciplinas de programação de computadores. Neste sentido, surge o Coding Dojo como uma alternativa metodológica para o ensino de algoritmos e programação de computadores que favorece o compartilhamento de experiências entre os participantes, aspectos de desafios em programação, interação e diversão [Bache 2013]. Pela postura dinâmica do Coding Dojo, acredita-se que aumentará a atenção dos alunos por meio da colaboração e atuará como um completemento das aulas tradicionais.

Este trabalho tem como objetivo geral expor as experiências na adoção da metodologia Coding Dojo em um curso de graduação de característica interdisciplinar. Com isso, almeja-se melhorar o processo de ensino/aprendizagem na programação de computadores e, como consequência, reduzir o índice de evasão no curso. Este documento está organizado da seguinte forma. A seção 2 comenta sobre e discute os trabalhos relacionados. Na seção 3, é apresentada a metodologia Coding Dojo e suas principais variações. A seção 4 apresenta a avaliação conduzida e a discussão dos resultados; e, finalmente, a seção 5 contém as conclusões e os direcionamentos futuros.

\section{Trabalhos Relacionados}

Luz e Serra-Seca-Neto (2012) apresentam características de Coding Dojo que favorecem o ensino de técnicas de desenvolvimento ágil para grupos de profissionais e estudantes de características e níveis heterogêneos [Luz and Serra-Seca-Neto 2012]. A metodologia utilizada no trabalho foi guiada pela aplicação de encontros de Coding Dojo e registros de atas dos encontros, destacando pontos positivos e negativos. Além disso, foi disponibilizado um questionário eletrônico respondido por participantes de diversas regiões do Brasil e com diferentes níveis de experiência. Os resultados experimentais apontam que os participantes concordam que Coding Dojo ajuda no aprendizado de métodos ágeis e a troca de experiências entre os participantes.

Delgado et al. (2012) discutem os pontos de convergência e divergência entre 
a metodologia Coding Dojo e as necessidades do ensino superior [Delgado et al. 2012]. Além disso, o trabalho propõe modificações do Coding Dojo para sua utilização em diferentes disciplinas dos cursos de graduação em Computação. Os autores afirmam que empregaram as modificações propostas em suas disciplinas que utilizam programação de computadores. No entanto, não houve uma melhor exploração do ganho alcançado pelas modificações, já que as modificações estão em estágio de maturação. Como trabalhos futuros, os autores pretendem fazer uma melhor compilação dos problemas para que as sessões de Coding Dojo sejam adequadas ao conteúdo das disciplinas de graduação em Computação.

Carmo e Braganholo (2012) estudam o uso didático da metodologia Coding Dojo como auxílio em disciplinas de graduação [Carmo and Braganholo 2012]. Para isso, foram especificadas diferentes dinâmicas de aulas baseadas em Coding Dojo e aplicadas a uma disciplina do curso de Ciência da Computação da Universidade Federal Fluminense (UFF). As dinâmicas procuravam ampliar o tempo para as discussões e motivar a participação dos alunos. Além disso, foram criados três padrões de aulas práticas, chamadas e Dojos individuais, Dojos em grupos e Laboratório em sala. Na avaliação experimental do trabalho, os autores elaboraram questionários aos quais os alunos respondiam após as sessões de Coding Dojo em suas diferentes dinâmicas. De acordo com os resultados, o padrão de Laboratório em sala foi a abordagem mais aceita pelos participantes.

Pode-se perceber que os trabalhos relacionados aplicaram a metodologia Coding Dojo em cursos ou disciplinas da área de tecnologia, em particular, na área da computação. Um curso de computação é caracterizado por possuir várias disciplinas onde os assuntos permeiam entre matemática e programação de computadores. Neste sentido, os alunos convivem com esses assuntos em todo o percurso do curso de graduação. No entanto, surge a necessidade de explorar a aplicação do Coding Dojo para um público de característica interdisciplinar, onde uma parte desse público não é voltado totalmente para a área de programação.

\section{Metodologia Coding Dojo}

O Coding Dojo consiste em uma metodologia que propõe encontros para que pessoas ganhem prática e conhecimento em alguma tecnologia previamente determinada, como programação [Bache 2013]. Nesse encontro é definido um mestre, que geralmente é o mais experiente com a tecnologia a ser abordada. O mestre possui a função de organizar o tempo e espaço para a dinâmica. Além disso, o mestre prepara desafios, não necessariamente complexos, antes do encontro e os propõe aos demais participantes. É comum que em determinados momentos alguns participantes não estejam resolvendo os desafios, isso pois estarão monitorando o trabalho de outros participantes, de modo a poder sugerir melhorias ou refletir sobre o que está sendo feito. Uma sessão de Dojo deve minimizar os erros de seus participantes a fim de que esses se sintam em um ambiente seguro. A competitividade não deve ser incentivada, e sim a colaboração e a troca de conhecimentos entre participantes. Dessa forma, é buscada a evolução dos participantes no que diz respeito às suas competências relacionadas às tecnologias abordadas [Heinonen et al. 2013].

Existem três formas de se aplicar uma sessão de Coding Dojo: Randori, Kata e Kake. Essas formas divergem entre si quanto a forma de se organizar e de se aplicar, entretanto possuem, em linhas gerais, o mesmo objetivo final, que consiste em aprender 
V Congresso Brasileiro de Informática na Educação (CBIE 2016)

Anais dos Workshops do V Congresso Brasileiro de Informática na Educação (CBIE 2016)

e treinar um grupo de pessoas a respeito de algum conceito ou tecnologia. A ideia de aprendizagem colaborativa também é um forte conceito possível a todas as formas supracitadas. Em sessões do tipo Randori, destacada pela Figura 1, um computador é colocado na frente da sala e sua tela é projetada para que todos possam ver. Nesse modelo os participantes exercem um dos seguintes papéis por vez: um piloto, que é o único que escreve o código no momento, um copiloto, que apoia e observa o piloto, e os demais participantes atuam como público, que observa e pode participar das discussões.

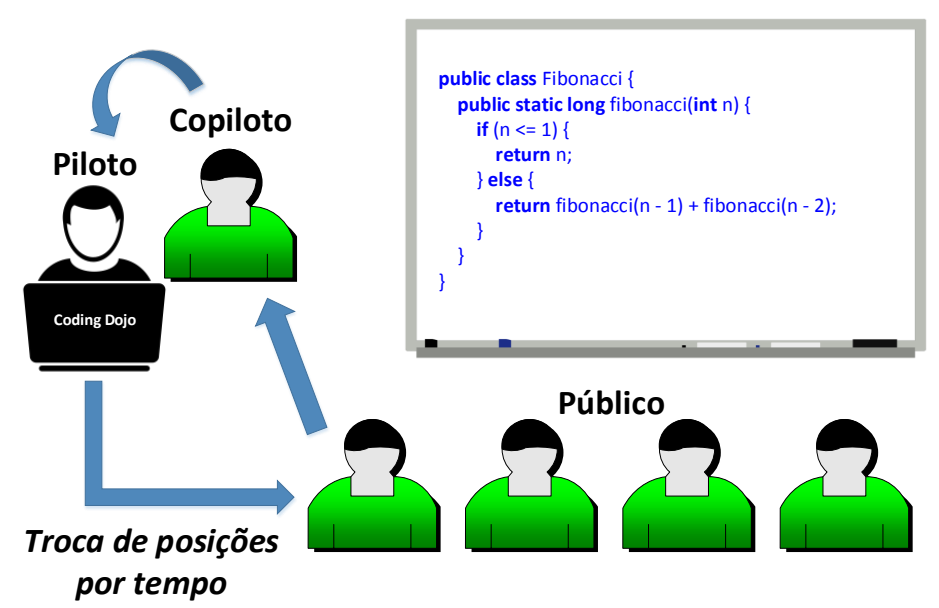

Figura 1. Randori Dojo

Uma característica comum é a troca de posições de tempo em tempo, sendo aconselhada a troca de posições num tempo que varie de cinco a sete minutos. A cada troca, $o$ piloto passa a fazer parte do público, o atual copiloto passa a ser o novo piloto e alguém do público passa a ser o novo copiloto. É fortemente recomendado que em pelo menos uma vez cada participante do processo exerça o papel de piloto e por pelo menos uma vez exerça também o papel de copiloto. Também é preciso que não haja um ambiente competitivo entre os participantes e sim a aprendizagem de modo que todos participem [Estácio et al. 2016].

O modelo Kata, ilustrado na Figura 2, possui foco no aperfeiçoamento. É sugerido que o problema proposto não seja longo, a fim de que possa ser resolvido em uma ou duas horas. O cerne desse modelo não é a resolução de um problema, e sim a qualidade da solução apresentada. Por esse motivo se sugere fortemente a repetição dos desafios, pois possibilitam o aperfeiçoamento da solução a cada resolução. Durante a repetição do desafio, pode ser mudado o paradigma de programação utilizado, por exemplo: o desafio já desenvolvido com Programação Orientada a Objetos pode ser resolvido posteriormente com Programação Funcional [Bache 2013].

Existem diversas vertentes utilizadas para o modelo Kata. Entretanto aqui será abordada a chamada Prepared Kata, que consiste em um ou mais participantes já terem resolvido o desafio anteriormente e fazerem novamente o desafio para apresentarem aos demais presentes, que podem fazer perguntas sobre. O objetivo é que os participantes depois possam resolver o mesmo problema. Aqui também pode ser aplicada a ideia de pares presente no Randori, de modo que a dupla possa trocar informações, o que causa maior troca de conhecimentos [Heinonen et al. 2013]. 
V Congresso Brasileiro de Informática na Educação (CBIE 2016)

Anais dos Workshops do V Congresso Brasileiro de Informática na Educação (CBIE 2016)
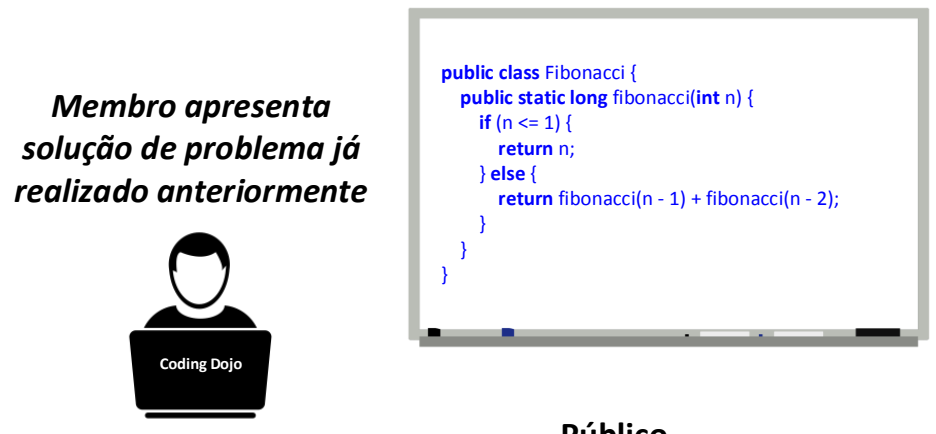

Público pode participar ou apenas assistir

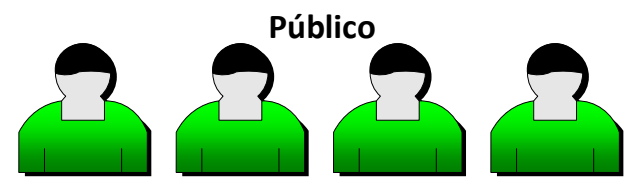

Figura 2. Kata Dojo

O modelo Kake, ilustrado na Figura 3, tem como diferencial a existência de diversas duplas trabalhando de forma concomitante e que podem ter alguma distinção entre elas. Essas distinções podem estar associadas ao uso de diferentes linguagens de programação para cada dupla [Cantone and Marchesi 2014]. Também é possível que cada dupla resolva um desafio dessemelhante da outra. Ao final de um turno, o piloto de uma dupla passa a ser o copiloto de uma outra dupla. Caso isso não seja possível por algum motivo, como a existência de números pares de participantes, o piloto se torna público e aguarda para se tornar copiloto no turno posterior [Luz 2013].

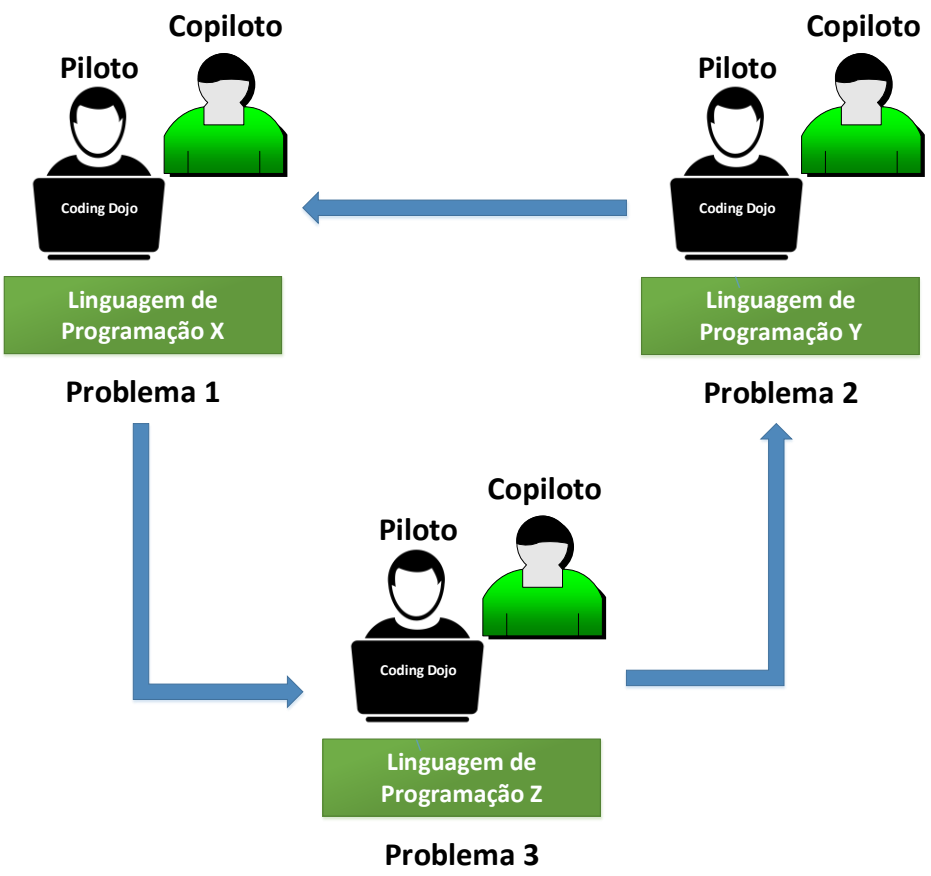

Figura 3. Kake Dojo

Um ponto a ser discutido no modelo Kake é que, por ter mais de uma dupla, não há 
V Congresso Brasileiro de Informática na Educação (CBIE 2016)

Anais dos Workshops do V Congresso Brasileiro de Informática na Educação (CBIE 2016)

uma centralização da atenção para um membro que resolve um desafio para todos verem. A descentralização da atenção pode ser um ponto positivo, uma vez que os participantes podem não se sentir pressionados para resolver um desafio enquanto todos da sala os observam.

\section{Avaliação}

A avaliação conduzida tem como objetivo verificar as melhorias alcançadas pela adoção da metodologia Coding Dojo em um curso com público de natureza interdisciplinar. Como instrumentos de avaliação utilizou-se um questionário a ser respondido pelos alunos participantes das sessões de Dojo, as notas/rendimento acadêmico dos alunos que participaram dos Dojos e as atas produzidas durante as sessões de Dojo. Como escopo temporal de observação e amostra, levou-se em consideração os alunos que participaram dos Dojos e regularmente matriculados na disciplina de Programação I do semestre de 2016.1 no curso de Sistemas e Mídias Digitais da Universidade Federal do Ceará. A turma (A) possuía 28 alunos, a turma (B) possuía 26 alunos. Já as turmas (C) e (D) são de alunos que foram reprovados na disciplina Programação I em semestres anteriores e possuíam, respectivamente, 19 e 16 alunos.

A forma de Dojo escolhida para a condução das sessões de Dojo no curso de Sistemas e Mídias Digitais foi o Kake. A justificativa para a adoção do Kake foi que é a forma que possibilita adaptação para utilizar diversas linguagens de programação em uma mesma sessão de Dojo. Nas duas turmas de repetentes foi adotada a linguagem C/C++. Já nas demais disciplinas foi utilizada a linguagem Processing. Com isso, as duplas podiam ser formadas por pessoas que utilizam a mesma linguagem de programação. Além disso, os problemas poderiam ser diferentes tanto em questões inerentes a própria linguagem, paradigma ou nível de complexidade. O Kake não necessita de divulgar para os demais participantes do Dojo como estão sendo resolvidos os desafios de programação. Com isso, diminuindo as chances dos alunos ficarem constrangidos e até intimidados ao expor suas soluções.

Os desafios de programação que foram lançados nas sessões de Dojos são referentes aos conteúdos da disciplina de Programação I. Ao todo, foram realizadas 6 sessões de Dojos, onde cada sessão era realizada de acordo com os avanços nos conteúdos das disciplinas durante o semestre. O conteúdo da disciplina de Programação I, até o semestre 2016.1, foi composto pelos seguintes tópicos: programação, sistemas e mídias digitais; conceitos de linguagens de programação; conceitos e estruturas básicas de algoritmos; programação estruturada: variáveis, operadores, escopo e bloco de comandos; álgebra Booleana; estruturas de seleção e repetição; sub-rotinas; vetores, matrizes e listas; noções de Programação Orientada a Objetos; práticas direcionadas através da construção de aplicações multimídia. A Tabela 1 expõe uma visão das sessões de Dojos que foram realizadas, total número de participantes e conteúdos dos desafios.

No total de sessões de Dojos realizados, houve a participação de 51 alunos, incluindo a contabilização da duplicidade de alunos que participaram de duas ou mais sessões de Dojo. Já levando em consideração a não duplicidade de alunos que participaram em duas ou mais sessões de Dojos, foram contabilizados 40 alunos. Para o total de alunos, juntando as quatro turmas de Programação I, foram contabilizados 89 alunos. Com isso, chegando a um percentual de $44,94 \%$ de alunos que participaram de alguma 
V Congresso Brasileiro de Informática na Educação (CBIE 2016)

Anais dos Workshops do V Congresso Brasileiro de Informática na Educação (CBIE 2016)

Tabela 1. Detalhamento das Sessões de Dojos Realizados no Semestre 2016.1

\begin{tabular}{l|l|l}
\hline Dojo & Participantes & Conteúdos dos Desafios \\
\hline \hline 1 & 6 & Lógica de Programação e Estruturas Condicionais \\
\hline 2 & 6 & $\begin{array}{l}\text { Estruturas Condicionais, Estruturas de Repetição e Ve- } \\
\text { tores }\end{array}$ \\
\hline 3 & 4 & $\begin{array}{l}\text { Estruturas Condicionais, Estruturas de Repetição e Ve- } \\
\text { tores }\end{array}$ \\
\hline 4 & 21 & $\begin{array}{l}\text { Estruturas de Repetição e Noções de Programação Ori- } \\
\text { entada a Objetos }\end{array}$ \\
\hline 5 & 8 & Estruturas Condicionais e Estruturas de Repetição \\
\hline 6 & 6 & $\begin{array}{l}\text { Estruturas de Repetição, Noções de Programação Ori- } \\
\text { entada a Objetos, Vetores e Matrizes }\end{array}$ \\
\hline
\end{tabular}

sessão de Dojo.

Com a observação do rendimento acadêmico dos alunos da turma (A) constatouse que 78,26\% alunos participantes do Dojo obtiveram aprovação na disciplina. O total participantes de Dojos da turma (A) foi de 23 alunos, onde 18 destes alunos foram aprovados. Uma visão geral do rendimento da turma (A) pode ser observada na Figura 4. Já na turma (B) contabilizou-se que $85,71 \%$ dos participantes de Dojos obtiveram aprovação na disciplina. A turma (B) teve um total de 7 alunos participantes, onde 6 destes alunos foram aprovados. A Figura 5 mostra o rendimento acadêmico obtido pela turma (B). Por fim, as turmas (C) e (D) tiveram 10 alunos, juntando ambas as turmas, participando de sessões de Dojos, onde 40\% deste total de alunos foram aprovados. Dos 10 alunos das turmas (C) e (D), 4 alunos obtiveram aprovação. Uma visualização gráfica do rendimento dos alunos das turmas (C) e (D) pode ser obtida na Figura 6. No geral e levando em consideração as quatro turmas, $70 \%$ dos alunos que participaram de alguma sessão de Dojo obtiveram a aprovação. O total de participantes de alguma sessão de Dojo foi de 40 alunos, onde 28 alunos alcançaram a aprovação.

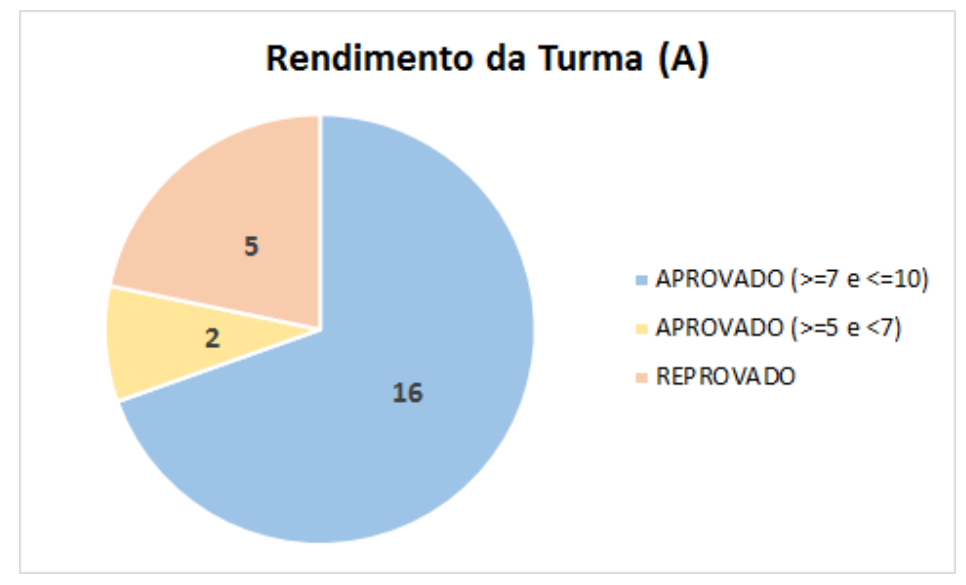

Figura 4. Rendimento da Turma (A)

Além da observação do rendimento acadêmico dos alunos participantes do Dojo, também foi elaborado um questionário para verificar a visão dos participantes no tocante 
V Congresso Brasileiro de Informática na Educação (CBIE 2016)

Anais dos Workshops do V Congresso Brasileiro de Informática na Educação (CBIE 2016)

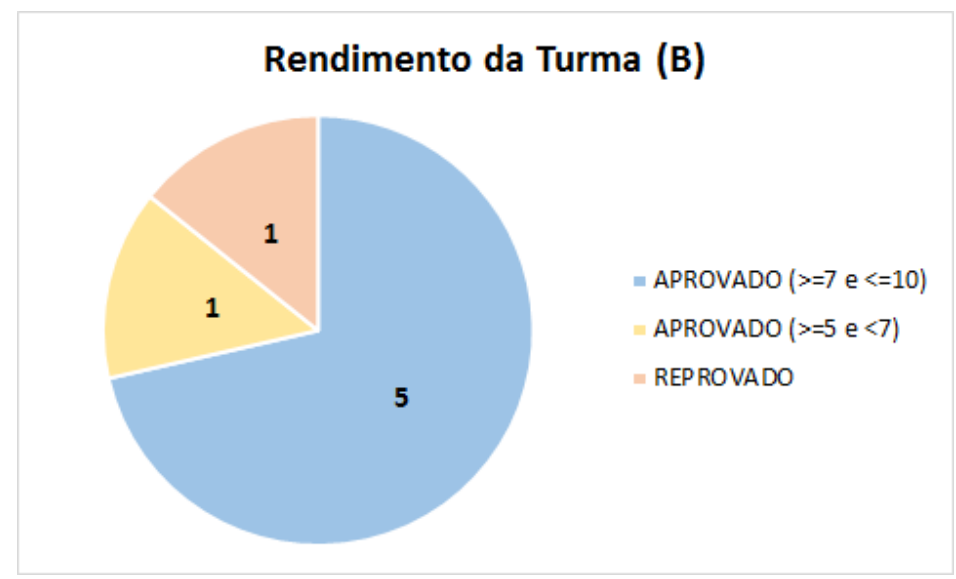

Figura 5. Rendimento da Turma (B)

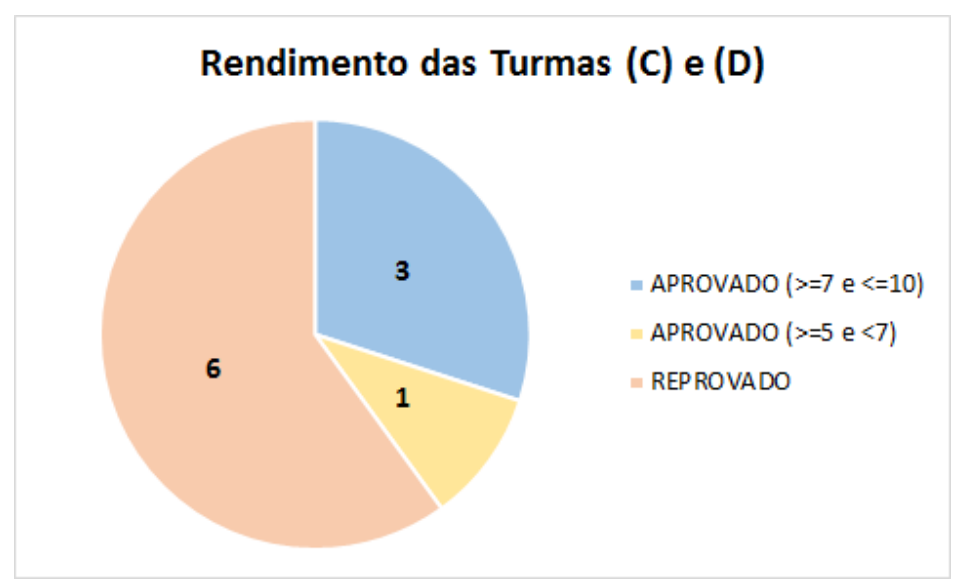

Figura 6. Rendimento das Turmas (C) e (D)

a metodologia Coding Dojo aplicada nos cursos de Sistemas e Mídias Digitais da UFC. A Tabela 2 exibe o conteúdo do questionário que foi disponibilizado aos alunos participantes de algum Dojo. Além disso, a Tabela demonstra a forma como as respostas podem ser aplicadas pelos participantes. Após o período de aplicação do questionário, contabilizouse as respostas de 10 alunos participantes de algum Dojo.

Dos 10 alunos que responderam ao questionário, 5 alunos eram da turma (A), 2 alunos eram da turma (B) e 3 alunos cursaram a turma (D). Com relação a segunda pergunta, 9 alunos responderam não conheciam a metodologia Coding Dojo. Em observação a terceira pergunta, 3 alunos disseram que concordam complementamente que a metodologia Coding Dojo contribuiu para melhorar os conhecimentos ministrados na disciplina de Programação I. Ainda na terceira pergunta, 6 alunos ressaltaram que concordam que o Coding Dojo ajudou a melhorar os conhecimentos na disciplina de Programação I, apenas 1 aluno ressaltou que discorda completamente com a terceira pergunta. De acordo com as respostas da quarta pergunta, a média de participação de alunos em quantidades de Dojos foi de 1,8. A quinta pergunta tem como objetivo verificar se a forma de Dojo aplicada foi adequada as necessidades dos alunos e da disciplina. De acordo com as respostas dos alunos, 1 aluno respondeu que concorda totalmente e 8 responderam que concordam que forma de Dojo foi adequada. Apenas 1 aluno respondeu que discorda completamente com 
V Congresso Brasileiro de Informática na Educação (CBIE 2016)

Anais dos Workshops do V Congresso Brasileiro de Informática na Educação (CBIE 2016)

Tabela 2. Questionário Aplicado aos Alunos Participantes de Dojos no Semestre 2016.1

\begin{tabular}{c|l|l}
\hline & Pergunta & Opçães de Resposta \\
\hline \hline 1 & $\begin{array}{l}\text { Qual foi o professor da sua turma de } \\
\text { Programação I no semestre 2016.1? }\end{array}$ & Texto aberto \\
\hline 2 & $\begin{array}{l}\text { Você já conhecia a metodologia Coding } \\
\text { Dojo? }\end{array}$ & $\begin{array}{l}\text { (a) Sim } \\
\text { (b) Não }\end{array}$ \\
\hline 3 & $\begin{array}{l}\text { Você concorda que a metodologia Co- } \\
\text { ding Dojo contribuiu para melhorar os } \\
\text { conhecimentos em ministrados na disci- } \\
\text { plina de Programação I? }\end{array}$ & $\begin{array}{l}\text { (a) Concordo completamente } \\
\text { (b) Concordo } \\
\text { (c) Discordo } \\
\text { (d) Discordo completamente }\end{array}$ \\
\hline 4 & $\begin{array}{l}\text { Quantos encontros de Dojos você fre- } \\
\text { quentou no semestre de 2016.1? }\end{array}$ & Número \\
\hline 5 & $\begin{array}{l}\text { Do modo como foi aplicada a metodolo- } \\
\text { gia Coding Dojo no curso de Sistemas e } \\
\text { Mídias Digitais, você concorda que faci- } \\
\text { litou o aprendizado nos assuntos minis- } \\
\text { trados na disciplina de Programação I? }\end{array}$ & (a) Concordo completamente \\
(c) Discordo \\
(d) Discordo completamente
\end{tabular}

quinta pergunta.

Já em relação a sexta pergunta, os pontos positivos mais recorrentes foram: "Metodologia Dinâmica", "Força o aluno a pensar", "Melhora o raciocínio lógico para programação" e "Estimula a prática em programação". Já em relação aos pontos negativos mais explicitados nas respostas da sétima questão foram: "Poderia ter um momento para explicação do código produzido por parte das equipes" e "Os desafios estavam simples demais nos primeiros Dojos". Por fim, a oitava questão é um complemento das demais, onde os alunos podem destacar algo a mais e de modo livre sobre sua experiência com o Coding Dojo. De acordo com os resultados, os pontos mais comentados pelos alunos foram: "Melhorar a divulgação dos Dojos", "Possibilidade de horários mais flexíveis para aplicação dos Dojos", "Realizar um maior número de sessões de Dojos e com menos desafios", "Melhorar o incentivo para a participação dos alunos" e "Aplicação de Dojos em outras disciplinas que envolvam programação".

\section{Conclusões e Trabalhos Futuros}

Este trabalho mostrou a aplicação de uma forma de Coding Dojo em um curso do ensino superior que possui um perfil de alunos de natureza interdisciplinar, ou seja, alunos que não são totalmente da área das ciências ou exatas. Além disso, foram discutidos as 
principais formas da metodologia Coding Dojo e alguns trabalhos correlatos. Por fim, foi descrito a avaliação do impacto da metodologia Coding Dojo em um curso interdisciplinar do ensino superior, destacando os resultados obtidos pelo rendimento acadêmico dos alunos participantes e um questionário para verificar o índice de aceitação da metodologia Coding Dojo por parte dos participantes. De acordo com os resultados da avaliação e as opiniões dos participantes, pode-se perceber que a metodologia Coding Dojo ajudou no desempenho dos alunos participantes, onde $70 \%$ destes alunos obtiveram aprovação na disciplina básica de programação. Ademais, alguns alunos foram consultados e, em geral, todos concordaram que a metodologia adotada na disciplina de Programação I foi satisfatória.

Como trabalhos futuros, pretende-se aplicar as outras formas de Coding Dojo e comparar os resultados entre as três formas apresentadas neste presente trabalho. Além disso, almeja-se adotar o Coding Dojo em outras disciplinas, que envolvem programação de computadores, do curso de Sistemas e Mídias Digitais da UFC. Com isso, verificar se houve um impacto positivo na evolução dos conhecimentos, no tocante a programação de computadores, dos alunos que participaram de Dojos em disciplinas anteriores. Neste presente trabalho constatou-se que 46,07\% dos alunos, matriculados em Programação I, participaram de alguma sessão de Dojo. Portanto, pretende-se melhorar a adesão dos alunos, seja por uma melhor divulgação, inserção de Dojos como atividades curriculares ou complementares do curso.

\section{Referências}

Bache, E. (2013). The Coding Dojo Handbook. Emily Bache, Canada.

Cantone, G. and Marchesi, M. (2014). Agile Processes in Software Engineering and Extreme Programming: 15th International Conference, XP 2014, Rome, Italy, May 2630, 2014, Proceedings. Lecture Notes in Business Information Processing. Springer International Publishing.

Carmo, D. H. and Braganholo, V. (2012). Um estudo sobre o uso didático de dojos de programação. In XX Workshop sobre Educação em Informática (WEI).

Delgado, C., Toledo, R., and Braganholo, V. (2012). Uso de dojos no ensino superior de computação. In XX Workshop sobre Educação em Informática (WEI).

Estácio, B., Zieris, F., Prechelt, L., and Prikladnicki, R. (2016). On the randori training dynamics. In Proceedings of the 9th International Workshop on Cooperative and Human Aspects of Software Engineering, CHASE '16, pages 44-47, New York, NY, USA. ACM.

Heinonen, K., Hirvikoski, K., Luukkainen, M., and Vihavainen, A. (2013). Learning agile software engineering practices using coding dojo. In Proceedings of the 14th Annual ACM SIGITE Conference on Information Technology Education, SIGITE '13, pages 97-102, New York, NY, USA. ACM.

Luz, R. B. (2013). A influência do dojo de programação no ensino de práticas ágeis. Master's thesis, Universidade Tecnológica Federal do Paraná, Curitiba, Paraná, Brasil.

Luz, R. B. and Serra-Seca-Neto, A. G. (2012). Usando dojos de programação para o ensino de desenvolvimento dirigido por testes. In $23^{\circ}$ Simpósio Brasileiro de Informática na Educação (SBIE). 